\title{
La ausencia de concordancia entre el clítico dativo y su referente nominal ${ }^{1}$
}

The absence of concordance between the dative clitic and its nominal referent

\author{
Vanina Andrea Barbeito \\ Julieta Murata Missagh \\ Silvina Peri
}

\section{Resumen}

El presente trabajo propone como tema de estudio la descripción de la relación de concordancia en número entre el objeto indirecto presente en la estructura oracional y el pronombre dativo para estudiar la duplicación de una forma plural con el pronombre átono singular le. En particular, nos ocupamos de la comparación de casos como (1) Les voy a bacer juicio a Twitter e Instagram, en los que se manifiesta concordancia en número entre el objeto y el pronombre, y (2) No levamos a crear problemas a los diplomáticos, en los que se evidencia ausencia de concordancia. El objetivo principal es aportar evidencia que permita indagar en un posible desgaste semántico del pronombre dativo, dado que el proceso de duplicación generalizada del objeto indirecto podría provocar que el clítico de dativo se vaya convirtiendo en una especie de morfema objetivo del verbo (Company, 2006) o de legitimador de un objeto oblicuo (Navarro, 2005), y le restaría valor como participante argumental (Torres Cacoullos, 2005). Esto provocaría la ausencia de concordancia de número entre el pronombre dativo singular y su referente nominal plural. En virtud de los principios del Enfoque, la descripción de las formas se realiza con atención a los contextos reales de aparición, y el análisis implica la medición de la significación estadística y representatividad de los datos a partir de pruebas objetivas. Por lo tanto, la metodología de este trabajo instrumenta un abordaje cualitativo y cuantitativo, a partir de la recolección de corpus de textos auténticos de la prensa escrita argentina de la actualidad. Se propone analizar el problema de la falta de concordancia con atención a aquellos factores lingüísticos que puedan condicionar la aparición de dicha estrategia, tales como la inanimación y posposición del objeto indirecto con respecto del verbo, y el número gramatical del objeto directo.

Palabras clave: objeto indirecto, pronombre dativo, concordancia, ditransitividad, referente

\begin{abstract}
This paper offers an insight on the description of the syntactic agreement between the singular dative pronoun and its nominal referent in a Spanish-written corpus. In particular, we focus on the comparison of examples such as (1) Les voy a hacer un juicio a Twitter e Instagram I'm going to sue Twitter and Instagram] and (2) No le vamos a crear problemas a los diplomáticos [We won't create problems for diplomats], the latter demonstrating lack of agreement. The main aim of this study is to offer evidence leading to a possible semantic depletion of the dative pronoun, given the fact that the process of generalized
\end{abstract}

1 El presente trabajo reúne resultados preliminares de un Proyecto de Reconocimiento Institucional de Investigadores Graduados (PRIG) de la Universidad de Buenos Aires. 
duplication of the indirect object may provoke that the dative clitic become a certain type of objective morpheme to the verb (Company, 2006) or a legitimizer of the oblique object (Navarro, 2005), and in turn undermine its value as argument participant (Torres Cacoullos, 2005). This would lead to the lack of agreement in number between the singular dative pronoun and its plural noun referent. Given such aim, we analyze whether the le pronoun and its noun referent are coreferential, with a view to determine if the lack of agreement furnishes evidence of a weakened referential ability of the dative pronoun. This paper seeks to examine if the clitic counts as an anaphor, in such cases in which an antecedent construction in agreement exists, or as an incorporated clitic, when no such agreement with the noun phrase serving as indirect object is offered. Following the principles of this theoretical perspective, linguistic forms are described through a careful attention to the real contexts of appearance, and their analysis includes the measurement of statistical significance and the accountability of the data through objective means. Therefore, this work methodology takes on a qualitative and quantitative approach, and resorts to the collection of corpora of authentic texts hailing from the present-day Argentinian written press.

Keywords: indirect object, dative pronoun, agreement, ditransitivity, referent

Vanina García Barbeito, Universidad de Buenos Aires, vanibarbeito@gmail.com

Julieta Murata Missagh, Universidad de Buenos Aires, julietamurata@gmail.com

Silvina Peri, Universidad de Buenos Aires, peri.silvina@gmail.com

Recibido: diciembre 2016 / Aceptado: abril 2017

DOI 10.17710/tep.2017.3.2.1barbeito

\section{Introducción. Fundamentos teóricos}

Uno de los rasgos distintivos del español, frente a otras lenguas romances, es la casi sistemática duplicación de un objeto indirecto con un clítico dativo correferencial dentro de la misma oración. En el presente trabajo indagaremos en la relación que se da entre el objeto indirecto presente en la estructura oracional y el pronombre que lo duplica para estudiar la relación de concordancia entre el pronombre dativo singular y su referente nominal plural sobre un corpus escrito del español. En particular, este trabajo se ocupa de la comparación de casos como (1) Les voy a hacer juicio a Twitter e Instagram, en los que se manifiesta concordancia en número entre el objeto y el pronombre, y (2) No le vamos a crear problemas a los diplomáticos, en los que se evidencia ausencia de concordancia.

Desde el enfoque teórico que nos enmarca, consideramos que la concordancia o la falta de ella está discursivamente motivada. En este sentido, analizamos factores influyentes en la ocurrencia del fenómeno como la inanimación y posposición del objeto indirecto (OI) respecto del verbo, y el número gramatical del objeto directo (OD). 


\section{Breve descripción del enfoque teórico}

\subsection{La concepción del lenguaje y la gramática}

La propuesta se inscribe en el marco del Enfoque Cognitivo Prototípico (Lakoff, 1987;Langacker, 1987), que considera que el conocimiento lingüístico integra el conocimiento del mundo y que la facultad del lenguaje se encuentra sujeta a las mismas reglas que el resto de las facultades. Al abandonar una visión modularista del lenguaje y plantear un único nivel de representación mental, el lenguaje se vuelve parte integrante de nuestra matriz psicológica general, de modo que toda explicación lingüística debe articularse con el conocimiento de los procesos cognitivos en general (Langacker, 1987: 13). Estos presupuestos tienen su correlato en una concepción distinta del signo lingüístico y del proceso de categorización, de la gramática, de la construcción del significado y del proceso de producción y comprensión de discursos.

El Enfoque Cognitivo-Prototípico (cfr. Lakoff 1987; Langacker 1987 y 1991, entre otros) concibe la categorización no como un producto arbitrario o un accidente histórico, sino como el resultado de principios psicológicos de categorización (Rosch, 1978). En este sentido, el proceso de categorización solo se conoce desde el uso, todo concepto es contextualmente dependiente, los criterios clasificatorios no son seleccionados arbitrariamente en beneficio de un sistema casi preestablecido, sino que se trata de develar los atributos más y menos salientes del elemento en cuestión a partir del presupuesto de que los atributos no deben estar todos presentes en todos los miembros de la categoría en la misma medida. Si las categorías representan mentalmente el conocimiento de la forma de uso de un objeto, el conocimiento tiene una estructura no arbitraria sino motivada por el uso del objeto en situaciones reales, y se sostiene así que los procesos lingüísticos responden a estrategias semejantes a las que rigen los otros procesos mentales, es decir, que no tienen naturaleza modular.

Desde este enfoque, se concibe la gramática como una colección abierta de estructuras o regularidades que provienen del discurso y toman forma a partir de él en un proceso permanente de reestructuración y resemantización en el uso (Hopper, 1998). En efecto, en el presente estudio adoptamos los lineamientos de una gramática emergente del discurso, un sistema en permanente (re)organización y sujeto a la convencionalización de rutinas lingüísticas exitosas. A diferencia de lo que sostienen otras perspectivas gramaticales que conciben la variación lingüística como alternancia libre de formas, para un enfoque que 
parte de la gramática emergente, la variación expresa un indicio de diferentes estrategias empleadas por los hablantes en el marco de determinadas constricciones contextuales.

Desde esta perspectiva, la gramática toma como postulado la motivación semántico-pragmática de los ordenamientos sintácticos y la sintaxis es síntoma del objetivo comunicativo, de aquello que el hablante quiere lograr o decir. En este sentido, las construcciones sintácticas deben ser analizadas necesariamente a la luz de los objetivos que se quieren lograr al usarlas y son más o menos prototípicas.

\subsection{La transitividad prototipica}

La teoría de prototipos se aplicó originalmente a la descripción de las posibilidades referenciales de los elementos léxicos (Rosch, 1978), aunque el interés del modelo se extendió también a la descripción semántica de la construcción transitiva. Al comparar las diferentes caracterizaciones, se observa un amplio consenso entre los autores en cuanto a las propiedades de la transitividad prototípica (Lakoff, 1977, p. 244; Langacker, 1991, p. 301-302).

Así, Taylor (1995, p. 206) ofrece un listado de rasgos semánticos de la construcción transitiva canónica que se enumeran sintéticamente a continuación: se describen eventos con dos participantes, codificados como sujeto y objeto directo; los dos participantes están claramente individualizados, son entidades contrapuestas y hay contacto físico entre ellos; el agente (sujeto) es humano, inicia el evento, actúa con conciencia y volición, y controla el evento; el paciente recibe los efectos de la acción realizada por el agente, y sufre un cambio de estado perceptible como consecuencia del evento; el evento es real, puntual y causativo. Las propiedades enumeradas por Taylor describen la semántica de un cierto tipo de construcciones transitivas, aquellas que se consideran prototípicas.

La construcción transitiva serviría a la expresión simbólica de la noción de causación. De ahí que las propiedades atribuidas a los casos paradigmáticos de causación directa (control, volición y responsabilidad del agente; cambio de estado físico perceptible en el paciente; contacto físico entre agente y paciente, etc.) coincidan a la perfección con los contenidos manifestados por las cláusulas transitivas prototípicas (Lakoff y Johnson, 1980, p. 70, Lakoff, 1977, p. 244).

Para representar el evento causal codificado a través de la construcción transitiva, se han elaborado diversos modelos compatibles entre sí dentro del marco de la Gramática Cognitiva. Langacker (1999: 24) se refiere al modelo de bola de billar como uno de los 
componentes del arquetipo conceptual correspondiente al modelo de evento canónico. Según este modelo conceptual idealizado, los objetos móviles que integran el mundo interaccionan con otros y les transfieren su energía.

Por su parte, Hopper y Thompson (1980, p. 254) conciben la transitividad como una relación central del lenguaje, que se expresa en una serie de consecuencias gramaticales de carácter predecible y cuyos rasgos definitorios están motivados discursivamente. A partir de una enumeración a priori de las situaciones que pueden designarse en cláusulas de al menos dos actantes, los autores definen la transitividad como el efecto de la relación entre diversos componentes semánticos, cuya gramaticalización da lugar a un ordenamiento gradual de cláusulas con mayor o menor transitividad relativa.

De manera más general, la concepción de la transitividad como la relación central de las gramáticas naturales acompaña la convicción de que sus componentes se hallan en un movimiento de covariación extensa y sistemática en el conjunto total de las lenguas. En ese sentido, los autores demuestran que todas las lenguas poseen estructuras morfosintácticas que reflejan variados grados de transitividad de la cláusula, una similitud que se fundamenta en la existencia de un marco de funcionamiento pragmático-discursivo que las contiene. En la puesta en uso del lenguaje, las instancias del discurso revelan una disposición cuidadosa de elementos: así, aquellas porciones de discurso que contribuyen de forma directa a los objetivos del hablante reciben el nombre de "primer plano", y aquellas que sirven como ampliación o comentario, son llamadas "fondo".

\section{Estado actual del conocimiento sobre el tema}

En el sistema de pronombres personales del español de América, el sistema de los clíticos de tercera persona lo, la y le se manifiesta siguiendo las pautas etimológicas y, en consecuencia, como un categorizador de caso y parcialmente de género. Sin embargo, tal como señala Martínez (2015), los sesgos de frecuencia de uso de las formas que se observan en distintas variedades de español americano, si bien superficialmente parecieran replicar pautas del español peninsular, configuran, por el contrario, sistemas propios. Por ejemplo, los desplazamientos de la forma le al campo de los acusativos, observado en distintas regiones, fueron alguna vez considerados "focos de leísmo" en América o bien aludidos como “neutralización de caso". Los análisis (García, 1986; Martínez, 2000) muestran que, a pesar de las distinciones intraparadigmáticas que se observan, en América se respeta el sistema de base caso; es decir, el paradigma etimológico. 
Por otra parte, a partir de la descripción de las diferencias en el empleo de clíticos, algunos estudiosos se han referido a una "confusión" en el uso de los clíticos, producto de las variedades no estandarizadas del español. Sin embargo, Martínez (2015, p. 190) señala que las diferencias observadas corresponden a desplazamientos intra-paradigmáticos del sistema etimológico que responden a necesidades comunicativas propias de la comunidad.

Una regla de concordancia del español es que los pronombres átonos deben concordar en número y persona con su referente, y para cierto tipo de pronombres también en género (Alarcos, 1994, pp. 198-199; RAE, 1973, \$2.5.2; entre otros). Sin embargo, el español suele quebrantar esta regla de concordancia, ya que el objeto indirecto plural suele ser duplicado con un pronombre singular. Company (2006, p. 544) indica que la estructura innovadora con pérdida de concordancia se halla desde hace siglos en variación con la estructura conservadora con mantenimiento de concordancia.

Basándose en Company (2002), Navarro (2005, p. 18) observa que el dativo ha sufrido diversos procesos que, a su vez, produjeron algunos cambios en el clítico, entre ellos la duplicación generalizada del OI. Este fenómeno tiene como efecto que el sintagma nominal dativo se vuelva central, argumental, mediante la agregación en la frase verbal de un clítico correferencial le que, según Company (2002, p. 51), se va convirtiendo en "una especie de morfema objetivo del verbo". Como consecuencia del proceso de duplicación y de la ausencia de la marca de pluralidad en el clítico, se produce un efecto de no concordancia, lo que va debilitando el estatus de pronombre anafórico del le. El clítico se convierte en una mera marca de concordancia objetiva para el verbo, "un marcador que anticipa en el verbo, como casi un afijo del verbo, que en ese evento sigue o existe un argumento" (Company 2002, p. 17).

Algunos autores (Wanner, 1987; Bresnan, 1998) sugieren que, en casos de doblado, el clítico es una marca morfológica de concordancia. Longa, Lorenzo y Rigau (1996) consideran que el rasgo de pluralidad generalmente se pierde en casos de doblamiento de dativo como en Voy a abrirle la puerta a los niños, pues el rasgo ya está codificado en el nominal los niños; pero en contextos donde no tengamos esta propiedad, este se codificará en el clítico. Por último, algunos autores van más allá y hablan de "incorporación pronominal" en aquellos casos en los que un pronombre clítico se afija al verbo convirtiéndose en un marcador morfológico de concordancia y posteriormente entra en un proceso de formación léxica. (Company, 2002; Suñer, 1986). 
Estudios recientes sobre diferentes dialectos del español (Company, 2006; Torres Cacoullos, 2005) sugieren que el pronombre de objeto indirecto ha sufrido desgaste semántico y que esto provocaría la ausencia de concordancia de número entre el pronombre dativo singular y su referente nominal plural.

En esta línea, Mojedano Batel (2014) analiza la falta de concordancia entre el pronombre dativo singular y su referente nominal plural en diferentes dialectos del español (México, España y Colombia), estudiando qué factores lingüísticos y sociales la favorecen. Concluye que aunque el pronombre dativo concuerde en número con su referente nominal plural en la mayoría de los casos, la falta de concordancia es un fenómeno frecuente condicionado por factores lingüísticos como la inanimación y posposición del OI con respecto del verbo, y el hecho de que el OD de la frase sea singular. Los resultados de su estudio lo llevan a concluir que el pronombre dativo situado antes del OI favorece la falta de concordancia, que los objetos inanimados promueven la falta de concordancia entre éstos y su referente pronominal, y que los OD singulares favorecen la falta de concordancia entre el OI nominal y su referente pronominal.

\section{Objetivos e hipótesis}

El objetivo principal del trabajo es aportar evidencia que permita indagar en un posible desgaste semántico del pronombre dativo. Se estima que el proceso de duplicación generalizada del OI podría provocar que el clítico de dativo se vaya convirtiendo en una especie de morfema objetivo del verbo (Company, 2006) o de legitimador de un objeto oblicuo (Navarro, 2005), y le restaría valor como participante argumental (Torres Cacoullos, 2005). Esto provocaría la ausencia de concordancia de número entre el pronombre dativo singular y su referente nominal plural.

En este sentido, se analiza la coincidencia de designado entre el pronombre le y su referente nominal para estudiar la duplicación de una forma plural con el pronombre átono singular le. Se busca definir si el clítico se comporta como anáfora, cuando hay un antecedente en concordancia, o como clítico incorporado, cuando no existe concordancia con el sintagma nominal que funciona como objeto indirecto.

De acuerdo con el estado del conocimiento sobre el tema, y con los fenómenos lingüísticos estudiados por los autores reseñados (cfr. \$3), se postulan las siguientes hipótesis: 
i) Los objetos antepuestos al verbo, generalmente más cercanos al pronombre dativo que los pospuestos, favorecen más la concordancia.

ii) El número gramatical del objeto directo puede afectar la falta de concordancia entre el pronombre y el nominal dativo: se espera mayor falta de concordancia cuando el OD sea singular.

iii) En ausencia de concordancia, le y el referente nominal plural OI no son realmente correferenciales. La presencia del pronombre le indica que en ese predicado existe un objeto indirecto, pero la falta de concordancia con su referente nominal plural es evidencia de que pierde su capacidad correferencial. Por eso, el término 'duplicación' no parece muy acertado en estos casos.

\section{Metodología y corpus}

Los presupuestos y criterios de descripción del enfoque adoptado, que postulan una gramática emergente del discurso, permiten hipotetizar el carácter original de nuestra descripción, al incorporar otros principios teóricos y al intentar contemplar todos los usos registrados efectivamente en la lengua. En este sentido, realizamos los trabajos de medición sobre un corpus auténtico del español y nuestro análisis implica la medición de la significación estadística y representatividad de los datos a partir de pruebas objetivas (Milroy, 1987).

Por lo tanto, la metodología de este trabajo instrumenta un abordaje cualitativo y cuantitativo, y se asienta sobre la recolección de un corpus de textos auténticos de la prensa escrita argentina de la actualidad. En la etapa actual del proyecto, el corpus se encuentra constituido por 151 ejemplos, provenientes de 135 artículos de 17 periódicos, tales como Clarin, La Nación y Página12, y publicados digitalmente entre 2015 y 2017.

En una primera aproximación al problema, nos interesa evaluar cuál es el contexto lingüístico en el que se produce el fenómeno de la ausencia de concordancia entre el pronombre dativo singular y su referente nominal plural. Para analizar este fenómeno, de los parámetros trabajados por otros autores, en esta oportunidad abordamos los de inanimación y posposición del objeto indirecto con respecto del verbo, y el número de la frase objeto directo. 
Se midió entonces la presencia y ausencia de concordancia entre el pronombre dativo y el sintagma nominal objeto indirecto sobre el total del corpus y en relación con los siguientes parámetros:

1) Posición del OI, pospuesto al verbo o antepuesto al verbo.

2) Número del OD, singular o plural

3) $\mathrm{OI}+/$ - animado

La medición de los parámetros consistió en contabilizar en cada miembro del par la presencia/ausencia del parámetro en cuestión.

\section{Análisis: algunos resultados preliminares}

Al observar todos los datos $(\mathrm{N}=151)$, el $73,51 \%$ de los mismos $(\mathrm{N}=111)$ mostró concordancia del OI nominal plural y su referente pronominal, como se puede ver en los ejemplos (1) y (2). Sin embargo, el 26,49 \% de los pronombres $(\mathbf{N}=40)$ no mostró concordancia con su referente nominal, como se observa en los ejemplos (3) y (4).

(1) Milagro Sala les pidió "disculpas" a sus hijos

(2) Quebrado en Ilanto, Santiago Bal les pidió disculpas a Denise Dumas y Pía Shaw

(3) Scioli también le prometió un ministerio a los intendentes

(4) El fin de una era: el macrismo le dice adiós a los candidatos famosos

El análisis de los datos revela que aunque el pronombre dativo concuerde en número con su referente nominal plural en la mayoría de los casos $(73,51 \%)$, la falta de concordancia es un fenómeno frecuente $(26,49 \%)$ a analizar.

El primer factor significativo para la variación del pronombre dativo fue la posición del OI. Sobre el total de los ejemplos, se midió cuántos casos presentaban el objeto indirecto pospuesto al verbo y cuántos casos lo presentaban antepuesto al verbo (es decir, más cercano al pronombre dativo). A su vez, dentro de cada uno de los subgrupos (OI antepuesto/OI pospuesto) se estableció la cantidad de casos que mostraban concordancia o falta de concordancia. Los resultados se observan en la Tabla 1.

Tabla 1. Posición del OI en relación con el verbo

\begin{tabular}{|c|r|c|c|}
\hline & \multicolumn{1}{|c|}{ A } & \multicolumn{1}{|c|}{$\mathbf{A}^{\mathbf{}}$} & \\
\hline & OI Antepuesto & OI Pospuesto & Totales \\
\hline + Concordancia & $43,58 \%$ & $56,41 \%$ & $100 \%$ \\
\hline B & 51 & 66 & $\mathbf{1 1 7}$ \\
\hline
\end{tabular}




\begin{tabular}{|c|c|c|c|}
\hline & $96,22 \%$ & $67,34 \%$ & \\
\hline - Concordancia & $5,26 \%$ & $94,11 \%$ & $100 \%$ \\
\hline \multirow[t]{2}{*}{ B' $^{\prime}$} & 2 & 32 & 34 \\
\hline & $3,7 \%$ & $32,29 \%$ & \\
\hline \multirow[t]{2}{*}{ TOTAL } & 53 & 98 & 151 \\
\hline & $100 \%$ & $100 \%$ & \\
\hline
\end{tabular}

Una lectura horizontal de la tabla muestra que el atributo [+] OI pospuesto favorece la falta de concordancia: el 94,11\% de los casos de no concordancia se dan con OI pospuesto, como se observa en los siguientes ejemplos:

(5) Scioli también le prometió un ministerio a los intendentes

(6) El fin de una era: el macrismo le dice adiós a los candidatos famosos

Por el contrario, la lectura vertical de la tabla evidencia que la anteposición del OI favorece la concordancia, con un $96,22 \%$ de los casos con OI antepuesto que exhiben concordancia, como se observa en el siguiente ejemplo:

(7) A muchos vecinos salir de noche les genera temory piden mejoras

Es decir que la posición prepuesta del objeto, más cercana al pronombre dativo, favorece la concordancia entre el pronombre y su referente nominal. En coincidencia con los resultados obtenidos por Mojedano Batel (2014:89), el hecho de que el pronombre dativo aparezca después de su referente nominal desfavorece la falta de concordancia entre ambos. Por el contrario, cuando el pronombre dativo se sitúa antes del OI, la falta de concordancia se ve favorecida.

El segundo factor significativo analizado como posible condicionante de la variación del pronombre dativo fue el número gramatical del OD. Tal como señalamos en $\$ 4$, se cree que este factor puede afectar la falta de concordancia entre el pronombre y el nominal, dado que se espera mayor falta de concordancia entre ellos que cuando cuando el objeto directo sea plural.

Para poner a prueba esta hipótesis, se midió la presencia de OD singular y OD plural sobre el total de los ejemplos. A su vez, dentro de cada subgrupo (+OD singular / +OD plural) se midió cuántos ejemplos mostraban concordancia y cuántos no la mostraban. Los resultados obtenidos se observan en la Tabla $2^{2}$.

\footnotetext{
${ }^{2}$ Incluimos dentro de los OD singular a las cláusulas sustantivas encabezadas por que o icónicas y a los pronombres interrogativos que cumplen dicha función.
} 
Tabla 2. OD singular y OD plural en relación con la presencia/ausencia de concordancia

\begin{tabular}{|c|c|c|c|}
\hline & $\mathbf{A}$ & $\mathbf{A}^{\prime}$ & \\
\hline & OD singular & OD plural & Totales \\
\hline + concordancia & $73,87 \%$ & $26,13 \%$ & $100 \%$ \\
\hline $\mathbf{B}$ & 82 & 29 & 111 \\
\hline & $72,56 \%$ & $76,31 \%$ & \\
\hline - concordancia & $77,50 \%$ & $22,50 \%$ & $100 \%$ \\
\hline $\mathbf{B}^{\prime}$ & 31 & 9 & 40 \\
\hline & $27,43 \%$ & $23,68 \%$ & \\
\hline TOTAL & 113 & 38 & 151 \\
\hline & $100 \%$ & $100 \%$ & \\
\hline
\end{tabular}

El número gramatical del objeto directo no parece afectar la falta de concordancia entre el pronombre y el nominal dativo. Contra toda expectativa, el $73,87 \%$ de los casos de concordancia se dan en presencia de un OD singular, como se observa en los siguientes ejemplos:

(8) Los delincuentes les sustrajeron el dinero a los hombres, pero los vio un policía vestido de civil, quien se sospecha que estaba custodiando al empresario en su diligencia bancaria.

(9) Macri les reclamó a los gobernadores "responsabilidad" con el gasto.

En efecto, la lectura horizontal de la Tabla 2 muestra que tanto los casos con concordancia como los casos sin concordancia muestran en su mayoría OD singular (73,87\% y 77,50\%, respectivamente). A su vez, la lectura vertical de la tabla arroja que sobre el total de los casos con OD singular es más frecuente la presencia de concordancia que su ausencia $(72,56 \%$ frente a $27,43 \%$ ). El mismo fenómeno se produce en los casos con OD plural $(76.31 \%$ frente a $23,68 \%)$.

Los casos de ausencia de concordancia se reparten de modo equilibrado entre aquellos con OD singular y con OD plural, como se muestra en los ejemplos (10) y (11), respectivamente:

(10) Le darán un perfil más técnico a las ex "universidades $K$ ” recién creadas

(11) El papa Francisco le abre las puertas a los divorciados pero rechaza el matrimonio bomosexual. 
A diferencia de autores como Mojedano (2014, p. 90), quien señala que los OD singulares favorecen la falta de concordancia entre el OI nominal y su referente pronominal, en nuestro corpus tanto la lectura vertical como horizontal de la tabla muestran que [+] OD singular no es un atributo determinante para la ausencia de concordancia.

El tercer factor significativo condicionante de la variación del pronombre dativo fue la animación del OI. La teoría de la transitividad (Hopper y Thompson, 1980) indicaría que los referentes nominales inanimados darían lugar a menos casos de concordancia que los animados. Por eso, se midió la influencia del rasgo +/- animado en la manifestación de concordancia o ausencia de ella. Sobre el total del corpus se contabilizaron los casos con OI animado y OI inanimado. A su vez, dentro de cada subgrupo, se midió cuántos manifestaban concordancia y cuántos no lo hacían. Los resultados se exponen en la Tabla 3.

Tabla 3. OI +/- animado en relación con la presencia o ausencia de concordancia

\begin{tabular}{|c|c|c|c|}
\hline & A & $A^{\prime}$ & \\
\hline Tabla 4 & + OI animado & - OI animado & Totales \\
\hline \multirow[t]{2}{*}{ + concordancia } & $93,58 \%$ & $6,42 \%$ & $100 \%$ \\
\hline & 102 & 7 & 109 \\
\hline B & $74,45 \%$ & $50 \%$ & \\
\hline - concordancia & $83,33 \%$ & $16,67 \%$ & $100 \%$ \\
\hline \multirow[t]{2}{*}{$B^{\prime}$} & 35 & 7 & 42 \\
\hline & $25,54 \%$ & $50 \%$ & \\
\hline \multirow[t]{2}{*}{ Total } & 137 & 14 & 151 \\
\hline & $100 \%$ & $100 \%$ & \\
\hline
\end{tabular}

Una lectura horizontal de la tabla muestra una alta frecuencia de aparición de OI $[+]$ animados en esquemas ditransitivos, ya que del total de los casos $(\mathrm{N}=151), 137$ casos mostraron la presencia de un objeto animado, por sobre 14 casos que presentaron objetos inanimados.

El atributo no parece ser determinante para la presencia o ausencia de concordancia, dado que tanto en los casos de concordancia (ejemplo 12) como en los de 
ausencia de ella (ejemplo 13), la mayoría de las construcciones presentó OI [+] animado (93,58\% y 83,33\%, respectivamente).

(12) "Por favor, se lo pido por mi familia, voy a hacer las cosas bien", les suplicó a los jueces sin poder contener el llanto Julio Gamboa, un soldador oriundo de Cutral Co y de 32 años.

(13) Erwin Tumiri es uno de los seis sobrevivientes de la tragedia aérea del martes en Antioquia que le quitó la vida a 71 personas, incluidos los jugadores y técnicos del equipo brasileño Chapecoense.

Sin embargo, una lectura horizontal de la tabla muestra que la presencia del objeto animado motiva la concordancia con un alto nivel de frecuencia relativa (un 93,58\% de los casos), como se observa en los siguientes casos:

(14) "Péguenme un tiro, ya está", les dijo a sus secuestradores.

(15) El diario de Báez, les sugiere a sus empleados que se autodespidan.

A diferencia de los resultados obtenidos por Mojedano Batel (2014), quien señala que los objetos inanimados promueven la falta de concordancia entre éstos y su referente pronominal, en nuestro corpus las construcciones con objeto inanimado mostraron presencia y ausencia de concordancia en partes iguales (50\% y 50\%), tal como se observa en los ejemplos (16) y (17), respectivamente:

(16) Frigerio les pone límites a los reclamos de los gremios.

(17) Le tapamos la boca a las críticas.

\section{Conclusiones preliminares}

Del análisis de los atributos medidos se concluye que la posición del objeto indirecto, antepuesto o pospuesto al verbo, es determinante para la presencia o ausencia de concordancia entre el pronombre dativo singular y su referente nominal plural. Los objetos antepuestos al verbo, generalmente más cercanos al pronombre dativo que los pospuestos, favorecen más la concordancia. El hecho de que el objeto indirecto pospuesto favorezca más la falta de concordancia entre el objeto y su referente nominal nos conduce a sostener la idea de que mientras mayor sea la distancia entre el objeto indirecto y el pronombre mayor será la tendencia a duplicar el objeto plural con un pronombre dativo singular. En general, cuando se presenta una anteposición del objeto indirecto, hay menor distancia léxica entre éste y el pronombre, mientras que cuando se pospone, hay mayor distancia entre ambos y crece la posibilidad de usar un pronombre singular para un referente dativo plural. 
Por otra parte, el contexto lingǘstico de uso muestra que el número del objeto directo no es un atributo determinante para favorecer la ausencia de concordancia. Es decir, que no se comporta como un factor significativo condicionante de la variación del pronombre dativo es la duplicación de un objeto indirecto con un clítico dativo correferencial dentro de la misma oración.

Con respecto a la animación del objeto indirecto, los datos mostraron que hay una alta frecuencia de aparición de objetos indirectos animados en esquemas ditransitivos y que la animación tiene un peso relativo para la ausencia de concordancia. La teoría de la transitividad (Hopper y Thompson, 1980) se ve respaldada por estos resultados, los cuales muestran que los OI animados tienden a concordar con su referente pronominal en la gran mayoría de los casos ya que parece que los hablantes los entienden como parte de una oración más altamente transitiva que aquella donde los objetos dativos son inanimados.

A partir de estos resultados, en próximos trabajos se analizará si el término "duplicación" es acertado en los casos de falta de concordancia, dado que no queda claro si el pronombre y el objeto son realmente correferenciales. En casos de duplicación, la presencia del pronombre le indicaría que en ese predicado existe un objeto indirecto, pero la falta de concordancia con su referente nominal plural sería evidencia de que pierde su capacidad correferencial.

\section{Referencias}

Alarcos Llorach, E. (1994). Gramática de la lengua española. Madrid: Espasa Calpe.

Bresnan, J. (1998). Markedness and Morphosyntactic Variation in Pronominal Systems. Handout for the Workshop Is Syntax Different? Common cognitive structures for syntax and phonology in Optimality Theory.12-13 de diciembre de 1998, Center for the Study of Language and Information.

Company Company, C. (2001). Multiple dative-marking grammaticalization: Spanish as a special kind of primary object language. Studies in Language 25, 1-47.

Company Company, C. (2002). Reanálisis en cadena y gramaticalización. Dativos problemáticos en la historia del español. Verba. Anuario Galego de filoloxía 29, 31-69.

Company Company, C. (2006). El objeto indirecto. En C. Company Company (Ed.), Sintaxis histórica de la lengua española. Primera parte: La frase verbal (Vol. I, pp. 479574). México: Fondo de Cultura Económica.

García, E. (1975). The Role of Theory in Linguistic Analysis: The Spanish Pronoun System. Amsterdam: North-Holland Publishing Company.

García, E. (1986). The case of Spanish gender: Referential strategies in language change.Neuphilologische Mitteilungen, 87, 165-184.

García, E. (1990). Bilingüismo e interferencia sintáctica. Lexis. XIV. 2. 159-195.

García, E., Otheguy, R. (1983). Being polite in Ecuador. Lingua, 61, 103-132. 
Hopper, J. P. (1998). Emergent Grammar. En M. Tomasello (ed.), The new psychology of language. Cognitive and functional approaches to language structure (pp. 155- 175). New Jersey: LEA.

Hopper, P. and S. Thompson (1980). Transitivity in grammar and discourse. Language 56.2:251-299

Klein-Andreu, F. (1993). Distintos sistemas de empleo de le, la, lo. Perspectiva sincrónica, diacrónica y sociolingüística. En O. Fernández Soriano (ed.). Los pronombres átonos (pp. 337-353). Madrid: Taurus.

Lakoff, G. (1977). Linguistic gestalts. Chicago Linguistic Society 13: 236-87.

Lakoff, G., (1987). Women, fire and dangerous things. Chicago: University Press.

Langacker, R (1991) Foundations of Cognitive Grammar. Descriptive Applications. Stanford: Stanford University Press, Vol.2.

Langacker, R.(1987). Foundations of Cognitive Grammar, vol. 1. Stanford: Stanford University Press.

Langacker, R. (1999). Grammar and Conceptualization.Cognitive Linguistic Research: 14. Berlin: New York, De Gruyter Mouton.

Longa, V., Lorenzo, G., \& Rigau, G. (1996). Expressing Modality by Recycling Clitics. Catalan Working Papers in Linguistics 5/1, pp. 67-79

Martínez, A. (2000). Lenguaje y Cultura. Estrategias etnopragmáticas en el uso de los pronombres cliticos lo, la y le en la Argentina en zonas de contacto con lenguas aborigenes(Tesis doctoral).Holanda: Universidad de Leiden, Instituto deLingüística Comparada.

Martínez, A. (2015) ¿Cómo afecta la cultura a la gramática? El caso de los clíticos en el español americano. Círculo de Lingüistica Aplicada a la Comunicación 61, 186-210.

Mauder, E. (2008) Pronoun variation in Latin American Spanish. A data engineer's view on le and lo. Netherlands: LOT.

Milroy, L. (1987). Observing and analyzing natural language. A critical account of sociolinguistic method. New York: Blackwell.

Mojedano, Andrea (2014) Variación de le/les en diferentes zonas hispanoparlantes: México, Colombia y España. IULC Working Papers, Vol.14: 2, Indiana University.

Navarro, I. (2005). Valores de le en español mexicano: un caso de incorporación pronominal. Barcelona: Universidad Autónoma de Barcelona.

Real Academia Española. (1975). Esbozo de una nueva gramática de la lengua española.Madrid: Espasa Calpe.

Rosch, E. (1978). Principles of categorization. In Eleanor Rosch y Barbara Lloyd (ed.). Cognition and categorization (pp. 27-47). Hillsdale, NJ: Erlbaum.

Suñer, M. (1986). Los Pronombres Nulos. Revista Argentina de Lingü̈stica 2, pp. 151-66.

Taylor, J. (1995). Linguistic Categorization. Prototypes in Linguistic Theory. Oxford University Press, USA.

Torres Cacoullos, R. (2005). La perspectiva diacrónica en variación sincrónica: el dativo de intensificación. En G. Knauer, \& V. Bellosta von Colbe (Eds.), Variación sintáctica en español: un reto para las teorias de la sintaxis (pp. 191-210). Tübingen: Niemeyer.

Wanner, D. (1987). Clitics Pronouns in Italian: al linguistic guide. Italica 64- 3, pp. 410-442.

Vanina Andrea Barbeito es Licenciada en Letras (UBA), Profesora de Enseñanza Media y Superior en Letras (UBA) y Periodista (TEA). Es actualmente doctoranda de la Facultad de Filosofía y Letras (UBA). Su tema de tesis es el análisis pragmático-semántico, sintáctico y 
prosódico de la construcción apositiva, problemática que aborda desde el Enfoque Cognitivo - Prototípico. Es Profesora Adjunta Regular de la cátedra Gramática en la Facultad de Filosofía y Letras (UBA) y directora del proyecto de investigación PRIG "La alternancia del pronombre dativo (le/les) en esquemas ditransitivos: referencia al objeto y proceso de desemantización", radicado en la misma institución. Es también investigadora en proyectos UBACyT y del Consejo Nacional de Investigaciones Científicas y Técnicas (CONICET).

Silvina Peri es Licenciada en Letras (UBA) y Profesora de Enseñanza Media y Superior en Letras (UBA). Actualmente es Ayudante de Trabajos Prácticos en Gramática en la Facultad de Filosofía y Letras (UBA); y subdirectora del proyecto de investigación PRIG "La alternancia del pronombre dativo (le/les) en esquemas ditransitivos: referencia al objeto y proceso de desemantización", radicado en la misma institución. Es profesora de la materia Lectura, escritura y oralidad del Profesorado de Inglés, en el Instituto Superior del Profesorado Dr. Joaquín V. González; y de Lengua y Literatura en instituciones de nivel Medio de la ciudad de Buenos Aires. Sus investigaciones se centran en el área de Gramática y consideran como marco teórico el Enfoque cognitivo prototípico.

Julieta Murata Missagh es Licenciada en Letras y Profesora de Enseñanza Media y Superior en Letras (Universidad de Buenos Aires), así como Traductora Técnica y Pública en Inglés (Universidad Nacional de Lanús). Desde el año 2014, desempeña tareas de asistencia de investigación en la cátedra de Gramática en la Facultad de Filosofía y Letras (UBA). Es integrante del proyecto PRIG titulado "La alternancia del pronombre dativo (le/les) en esquemas ditransitivos: referencia al objeto y proceso de desemantización". Ha participado de varios eventos académicos nacionales e internacionales en calidad de expositora, y ha publicado artículos de investigación en las áreas de gramática y sociolingüística. 